\title{
Avaliação do uso da resazurina em teste de suscetibilidade in vitro frente a Sporothrix brasiliensis
}

\author{
Beatriz Mendes Roca ${ }^{\mathrm{a}}$, Vanice Rodrigues Poester ${ }^{\mathrm{a}, \mathrm{b}}$, Antonella Souza Mattei ${ }^{\mathrm{c}}$, \\ Gabriel Baracy Klafke ${ }^{\mathrm{a}}$, Ivy Bastos Ramis ${ }^{\mathrm{b}}$, Melissa Orzechowski Xavier ${ }^{\mathrm{a}, \mathrm{b}^{*}}$ \\ ${ }^{a}$ Laboratório de Micologia, Faculdade de Medicina, Universidade Federal do Rio Grande, Rio Grande, RS, Brasil \\ ${ }^{\text {b} P r o g r a m a ~ d e ~ P o ́ s-G r a d u a c ̧ a ̃ o ~ e m ~ C i e ̂ n c i a s ~ d a ~ S a u ́ d e, ~ F a c u l d a d e ~ d e ~ M e d i c i n a, ~}$ \\ Universidade Federal do Rio Grande, Rio Grande, RS, Brasil \\ ${ }^{\mathrm{c}}$ Universidade de Caxias do Sul - UCS, Caxias do Sul, RS, Brasil
}

Histórico do Artigo Recebido em: 12/04/2019

Aceito em: 29/08/2019

Palavras-chave: Indicador de crescimento; itraconazol; esporotricose; microdiluição em caldo

Keywords: Growth indicator, itraconazole, sporotrichosis, broth microdilution

\begin{abstract}
RESUMO
A técnica de microdiluição em caldo, que permite determinar a concentração inibitória mínima (CIM) dos fármacos frente aos microrganismos, é considerada padrão ouro para avaliação do perfil de suscetibilidade fúngica in vitro. Considerando que a leitura dos resultados é realizada pela visualização do crescimento fúngico, e que a utilização de indicadores de crescimento microbiano poderia otimizar essa leitura, o estudo teve como objetivo avaliar o uso da resazurina como indicador do crescimento de Sporotrhix brasiliensis em testes de microdiluição em caldo. Cinco isolados clínicos (nas fases leveduriforme e filamentosa) foram testados frente ao itraconazol (ITC - concentrações de 0,0313 a $16 \mu \mathrm{g} / \mathrm{mL}$ ) a partir da técnica de microdiluição em caldo, utilizando a resazurina nas concentrações de $0,01 \%$ e $0,002 \%$. A leitura dos resultados foi realizada após 24, 48 e 72 horas de incubação, e a CIM dos poços com resazurina foi considerada como sendo a menor concentração sem modificação na coloração, enquanto que a CIM do controle, foi considerada a menor concentração de fármaco capaz de inibir o crescimento fúngico. Os resultados da mudança da cor da resazurina foram comparados com os resultados sem acréscimo de resazurina. A CIM do ITC na microdiluição clássica frente aos isolados testados variou de 0,0625 a $1 \mu \mathrm{g} / \mathrm{mL}$, porém, com o uso da resazurina como indicador de crescimento fúngico, independente da concentração testada, a CIM foi $\leq 0,0625 \mu \mathrm{g} / \mathrm{mL}$. O estudo evidencia que a resazurina não é eficaz como indicador de crescimento de $S$. brasiliensis visto que não modificou a coloração do meio de cultivo, mesmo na presença visível de crescimento fúngico, sendo portanto contra indicada para uso em testes de suscetibilidade in vitro frente a este microrganismo.
\end{abstract}

\section{Evaluation of the resazurin applied at in vitro susceptibility test against Sporothrix brasiliensis}

\begin{abstract}
The broth microdilution technique that allows determining drugs minimum inhibitory concentration (MIC) against microorganisms is considered the standard test to evaluate the fungal in vitro susceptibility. Considering the results are usually read through fungal growth and that the use of microbiological growth indicators can optimize the reading, this study had the objective to evaluate the use of resazurin as an indicator of Sporothrix brasiliensis growth in broth microdilution tests. Five clinical strains (on yeast and filamentous form) were tested against itraconazole (ITC - between 0.0313 to $16 \mu \mathrm{g} / \mathrm{mL}$ concentration) through broth microdilution technique, using the resazurin on concentrations of $0.01 \%$ and $0.002 \%$. The results were read after 24, 48 and 72 hours of incubation, and the MIC of the wells with resazurin was considered the lowest concentration without medium coloring modification by resazurin's reduction, while the control MIC was considered the lowest concentration capable of inhibit the fungal growth. These results were compared to the classical microdilution test's results, without adding resazurin. The ITC's MIC on classical microdilution against the tested isolates ranged from 0.0625 to $1 \mu \mathrm{g} / \mathrm{mL}$, however, using resazurin as a fungal growth indicator, regardless the tested concentration, the MIC was $\leq 0.0625 \mu \mathrm{g} / \mathrm{mL}$. The study shows that the resazurin is not effective as a growth indicator of $S$. brasiliensis because it didn't modified the growing medium's colour, even with visible fungal growth, therefore, being contraindicated for use in in vitro susceptibility tests against this microorganism.
\end{abstract}

\footnotetext{
*Autor para correspondência: melissaxavierfurg@gmail.com (Xavier M.O.)
} 


\section{Introdução}

A resistência antifúngica tem sido alvo de estudos, devido a importância que esta condição tem para o tratamento de micoses, gerando falhas terapêuticas $(1,2)$. A avaliação da resistência antifúngica é realizada a partir de testes de suscetibilidade in vitro de isolados fúngicos a diferentes fármacos, pela técnica de microdiluição em caldo $(3,4)$. Esta técnica permite determinar a concentração inibitória mínima (CIM) do fármaco, a qual é utilizada para classificar o isolado fúngico em sensível, sensível dosedependente ou resistente, de acordo com protocolos internacionalmente aceitos, Clinical and Laboratory Standards Institute e European Committee on Antimicrobial Susceptibility Testing (3-5). Essa classificação auxilia no tratamento correto de doenças fúngicas, evitando o uso indiscriminado de fármacos (6).

A determinação da CIM é realizada de forma visual, por comparação do crescimento fúngico ao controle de crescimento, e o tempo de incubação necessário até a leitura dos resultados oscila de 21 até 74 horas, de acordo com o gênero fúngico avaliado $(7,8)$. Nesse sentido, o uso de reagentes que atuem como indicadores de viabilidade celular vem sendo descrito com o intuito de auxiliar na leitura dos resultados dos testes de suscetibilidade in vitro, como é o exemplo do brometo de tetrazolio (MTT) e da resazurina, os quais apresentam mudança colorimétrica devido a uma reação de oxiredução (9-12).

A utilização da resazurina já vem sendo avaliada para fungos leveduriformes (Candida spp. e Malassezia spp.) e filamentosos (Aspergillus spp.), apresentando promissora aplicação para testes de suscetibilidade in vitro na área da micologia $(10,11,13)$. Este reagente, quando exposto a produtos oriundos da respiração celular microbiana, converte-se em resorufinapor uma reação de oxi-redução. Assim, a sua cor originalmente azul, passa à rosa, indicando crescimento microbiano (10-13).

No entanto, sua aplicação para testes de suscetibilidade in vitro frente a fungos do gênero Sporothrix ainda não foi descrita. Assim, tendo em vista que a esporotricose é uma micose subcutânea que vem se tornando um grave problema de saúde pública devido ao seu aumento de casos zoonóticos e felinos no Brasil, somado ao tempo de leitura de 72 horas para o antifungigrama do principal agente etiológico desta doença ( $S$. brasiliensis) $(14,15)$, este estudo objetivou avaliar a resazurina como indicador do crescimento de $S$. brasiliensis.

\section{Material e métodos}

Foram utilizados cinco isolados de $S$. brasiliensis provenientes de casos de esporotricose felina do município do Rio Grande, Rio Grande do Sul, Brasil. Estes isolados foram previamente identificados por técnicas de biologia molecular e encontravam-se estocados na micoteca do Laboratório de Micologia da Faculdade de Medicina da Universidade Federal do Rio Grande (FAMED - FURG). Os isolados selecionados foram determinados como sensíveis ao ITC $(\mathrm{CIM}<2 \mu \mathrm{g} / \mathrm{mL})$, conforme descrito por Espinel-Ingrof et al. (16).

Para os testes de microdiluição em caldo, as colônias foram subcultivadas em ágar batata (PDA) e em ágar Brain-Heart Infusion (BHI) e mantidas em estufas de $25^{\circ} \mathrm{C}$ e de $37^{\circ} \mathrm{C}$, durante 7 dias, para a obtenção da fase filamentosa e leveduriforme do fungo, respectivamente. Os inóculos foram preparados com solução salina estéril e padronizados em espectrofotômetro $(530 \mathrm{~nm})$ dentro do intervalo de absorbância de 0,09 e 0,13, de acordo com o CLSI (3). Esta solução foi diluída em RPMI 1640 na 
proporção 1:50, obtendo-se $0,8 \times 10^{4}$ a 1 × $10^{5}$ Unidades Formadoras de Colônia (UFCs) por mililitro, a qual corresponde a uma concentração duas vezes superior a concentração testada in vitro $\left(0,4 \times 10^{4}\right.$ a $\left.5 \times 10^{4} \mathrm{UFC} / \mathrm{ml}\right)$. A concentração do inóculo foi confirmada pela técnica de pour-plate em todos os testes realizados.

A microdiluição em caldo, na ausência e na presença da resazurina, foi realizada em microplacas de 96 poços, utilizando itraconazol (Prati-Donaduzzi ${ }^{\circledR}$ ) (ITC), nas concentrações de 0,0313 a $16 \mu \mathrm{g} / \mathrm{mL}$, diluído em RPMI, seguindo o protocolo M38-A2 do CLSI (3) para fase filamentosa, e M27-A3 adaptado (17) para a leveduriforme. Adicionalmente, em todas as microplacas foi acrescentado um controle de crescimento (meio de cultivo + inóculo) e dois controles de esterilidade (somente meio de cultivo; meio de cultivo + ITC).

A resazurina (Sigma-Aldrich ${ }^{\circledR}$ ) foi preparada em uma solução estoque na concentração de $0,02 \%$ em água destilada estéril e sua aplicabilidade como indicador de crescimento de $S$. brasiliensis foi avaliada por três metodologias distintas (Figura 1). Na primeira metodologia, a resazurina na concentração inicial de $0,002 \%$ foi adicionada em cada poço ao término da montagem da microplaca (11); na segunda metodologia, a resazurina, mais concentrada $(0,01 \%)$, foi adicionada diretamente ao inóculo padronizado previamente ao preenchimento da microplaca (10); e, na última metodologia, a resazurina $(0,002 \%)$ foi adicionada em cada poço da microplaca 24 horas antes da leitura dos resultados (12). As duas primeiras metodologias foram aplicadas tanto à fase filamentosa, quanto à fase leveduriforme do fungo, já a terceira foi testada apenas na fase filamentosa, por ser a fase protocolada para a realização desta técnica (3)

Para todas as metodologias avaliadas foram utilizados um controle de crescimento com adição de resazurina, e adicionalmente, em todas as microplacas foi realizada a microdiluição clássica, sem adição da resazurina, como controle para comparação dos resultados $(3,17)$.

Foi realizada leitura visual após 24,48 e $72 \mathrm{hs}$ de incubação a $25^{\circ} \mathrm{C}$ para os testes com a fase filamentosa e após $24,48,72,96$ e $120 \mathrm{hs}$ a $37^{\circ} \mathrm{C}$ para os testes com a fase leveduriforme, respectivamente. Na técnica padrão (avaliação visual do crescimento fúngico), utilizada como controle, a CIM foi aquela capaz de inibir $100 \%$ do crescimento fúngico; enquanto que nos testes com adição do reagente, a CIM foi considerada a menor concentração em que não houve mudança na coloração da solução, de azul para rosa. Ao final, os resultados de CIM do teste controle (microdiluição sem adição de resazurina) foi comparado com a CIM dos testes com adição da resazurina para avaliação da eficácia na utilização do reagente. Para fins de interpretação dos resultados, foi determinado como crescimento moderado e fraco a redução de $20-30 \%$ e $\geq 50 \%$, respectivamente, em comparação com o controle de crescimento.

\section{Resultados e discussão}

A CIM do grupo controle (sem adição de resazurina) variou de 0,125 a $1 \mu \mathrm{g} / \mathrm{mL}$ na fase filamentosa e de 0,0625 a $0,5 \mu \mathrm{g} / \mathrm{mL}$ na fase leveduriforme, o que condiz com os resultados expostos na literatura $(17,18)$. No entanto, a CIM utilizando a resazurina foi $\leq 0,0625 \mu \mathrm{g} / \mathrm{mL}$ em todas as metodologias avaliadas, sendo evidenciada apenas em poços com crescimento abundante de $S$. brasiliensis, controle de crescimento (sem ITC) ou poços com pouca concentração do fármaco (Figura 1). 
Figura 1 - Microdiluição em caldo do itraconazol frente a um isolado de S. brasiliensis, realizado em duplicata, demonstrando leveoxi-redução da resazurina (mudança de cor de azul para rosa somente nos poços com exuberante crescimento fúngico) em duas das três metodologias testadas no estudo.

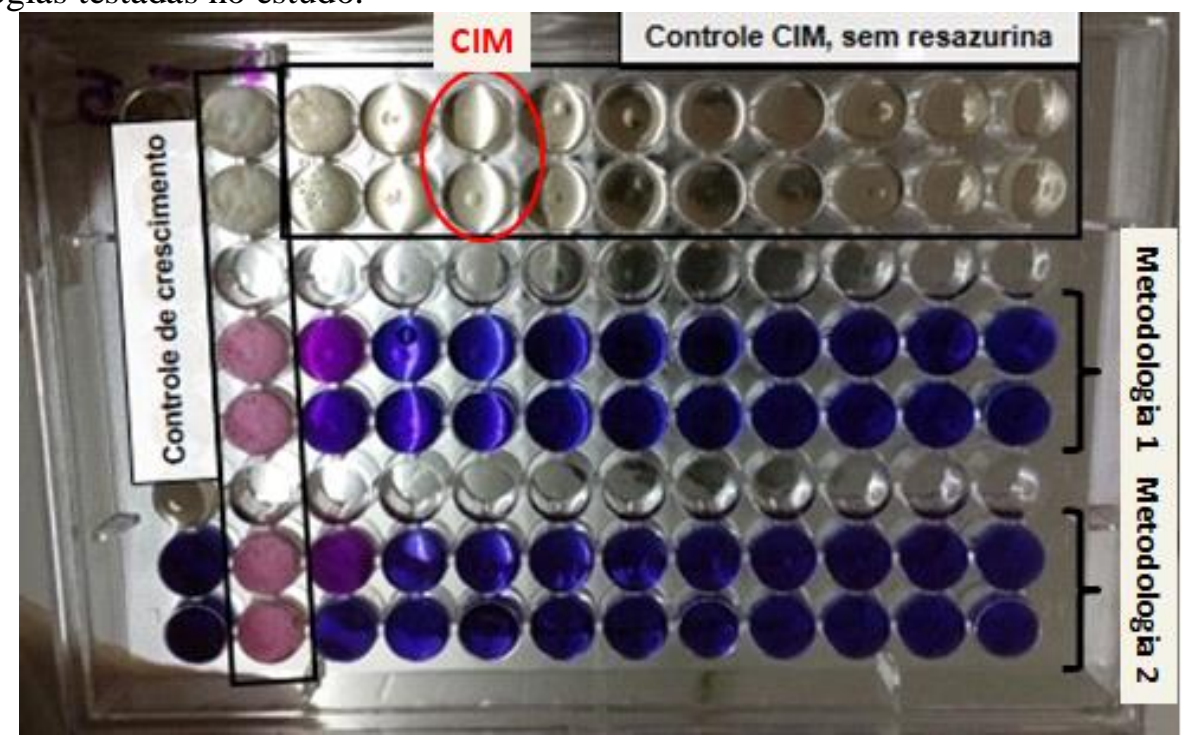

Na bacteriologia, a resazurina tem sido utilizada em testes de suscetibilidade, já sendo preconizada para otimização dos resultados frente a alguns microrganismos como, por exemplo, Mycobacterium spp., Neisseriagonorrhoeae e Chlamydiatrachomatis (12, 1921). Na área da micologia, o uso desse reagentevem sendo proposto, porém ainda com poucos gêneros fúngicos testados e sem padronização na sua utilização $(10,11,13)$.

$\mathrm{O}$ potencial da utilização da resazurina em testes de suscetibilidade in vitro para fungos já foi demonstrado frente aos gêneros Aspergillus, Malassezia e Candida (10, $11,13)$. No entanto, este reagente não se mostrou efetivo para avaliação da CIM em $S$. brasiliensis nas condições aplicadas no estudo, utilizando o ITC como fármaco, visto que não foi observada modificação da coloração do meio nos poços em que houve crescimento fúngico moderado e fraco. O mecanismo de ação da resazurina está relacionado à sua oxi-redução por produtos oriundos da respiração celular (10); no entanto, por fatores ainda a serem elucidados, o reagente só passou para sua forma reduzida com o crescimento abundante de $S$. brasiliensis.

Nossos resultados estão ao encontro do descrito por Monteiro et al. (11) que descrevem que a densidade fúngica interfere na ação da resazurina para indicar o crescimento fúngico, e que seria necessária uma concentração maior de inóculo do que aquela preconizada pelo CLSI para sua eficiência. Além disso, Monteiro et al. ressalta a utilização de RPMI-1640 modificado (10,4 g/L de RPMI-1640; 6,7 g/L de YNB; 1,8\% glicose e $40 \mathrm{mM}$ HEPES em $\mathrm{pH}=7.1$ ) para os testes com resazurina na suscetibilidade in vitro de Aspergillus spp. $\mathrm{O}$ presente estudo utilizou como meio de cultivo RPMI-1640 convencional, portanto estudos futuros podem ser conduzidos com as modificações descritas, com intuito de aprimorar a técnica para utilização com Sporothrix spp.

\section{Conclusões}

O estudo evidencia que nas condições testadas a resazurina não é eficaz como indicador de crescimento de $S$. brasiliensis visto que não houve modificaçãona coloraçãodos poços em que havia crescimento fúngico visível, portanto mais estudos 
são necessários para aprimoramento da técnica e/ou elucidação dos fatores intrínsecos de Sporothrix spp. possivelmente relacionados ao resultado encontrado.

\section{Agradecimentos}

Os autores são gratos a Coordenação de Aperfeiçoamento de Pessoal de Nível Superior (CAPES) e ao Conselho Nacional de Desenvolvimento Científico e Tecnológico (CNPQ) pela concessão de bolsas de estudo.

\section{Referências}

1. Bergold AM, Georgiadis S. Novidades em fármacos antifúngicos: uma revisão. Visão Acadêmica 2004; 5(2):159-72.

2. Perlin DS, Rautemaa-Richardson R, Alastruey-Izquierdo A. The global problem of antifungal resistance: prevalence, mechanisms, and management. Lancet Infect Dis 2017;17(12):e383-92.

3. CLSI. Reference Method for Broth Dilution Antifungal Susceptibility Testing of Filamentous fungi, approved standard, M38-A2. In: Wayne, editor. Clinical And Laboratory Standards Institute. 2nd ed. 2008.

4. Pfaller MA. Antifungal Drug Resistance: Mechanisms, Epidemiology, and Consequences for Treatment. Am J Med 2012;125(1, Supplement):S3-13.

5. EUCAST. EUCAST, Subcommittee on Antifungal Susceptibility Testing of the ESCMID European Committee for Antimicrobial Susceptibility Testing. Clin Microbiol Infect 2008;14:982-4.

6. Eggimann P, Lamoth F, Marchetti O. On track to limit antifungal overuse! Intensive Care Med 2009;35(4):582-4.

7. CLSI. Reference Method for Broth Dilution Antifungal Susceptibility Testing of Yeasts. Approv Stand M27-A2. 2002.

8. CLSI. Reference Method for Broth Dilution Antifungal Susceptibility Testing of Filamentous Fungi. Approv Stand M38-A. 2002.

9. Montoro E, Lemus D, Echemendia M, Martin A, Portaels F, Palomino JC. Comparative evaluation of the nitrate reduction assay, the MTT test, and the resazurin microtitre assay for drug susceptibility testing of clinical isolates of Mycobacterium tuberculosis. J Antimicrob Chemother 2005;55(4):5005.

10. Liu M, Seidel V, Katerere DR, Gray AI. Colorimetric broth microdilution method for the antifungal screening of plant extracts against yeasts. Methods 2007;42(4):325-9.

11. Monteiro MC, De La Cruz M, Cantizani J, Moreno C, Tormo JR, Mellado E, et al. A new approach to drug discovery: High-throughput screening of microbial natural extracts against Aspergillus fumigatus using resazurin. J Biomol Screen 2012;17(4):542-9.

12. Ramis IB, Cnockaert M, von Groll A, Nogueira CL, Leão SC, Andre E, et al. Antimicrobial susceptibility of rapidly growing mycobacteria using the rapid colorimetric method. Eur $\mathrm{J}$ Clin Microbiol Infect Dis 2015; 34(7):1403-13.

13. Leong C, Buttafuoco A, Glatz M, Bosshard PP. Antifungal susceptibility testing of Malassezia spp. with an optimized colorimetric broth microdilution method. J Clin Microbiol 2017;55(6):1883-93.

14. Rodrigues AM, Teixeira M de M, de Hoog GS, Schubach TMP, Pereira SA, Fernandes GF, et al. Phylogenetic analysis reveals a high prevalence of Sporothrix brasiliensis in feline sporotrichosis outbreaks. PLoS Negl Trop Dis 2013;7(6):e2281.

15. Poester VR, Mattei AS, Madrid IM, Pereira JTB, Klafke GB, Sanchotene KO, et al. Sporotrichosis in Southern Brazil, towards an epidemic? Zoonoses Public Health 2018; 65(7): 815-821.

16. Espinel-Ingroff A, Abreu DPB, Almeida-Paes R, Brilhante RSN, Chakrabarti A, Chowdhary A, et al. Multicenter, International Study of MIC/MEC Distributions for Definition of Epidemiological Cutoff Values for Sporothrix Species Identified by Molecular Methods. Antimicrob Agents Chemother 2017; 61(10): e01057-17.

17. Sanchotene KO, Brandolt TM, Klafke GB, Poester VR, Xavier MO. In vitro susceptibility of Sporothrix brasiliensis: Comparison of yeast and mycelial phases. Med Mycol 2017;55(8):869-76. 
18. Poester VR, Mattei AS, Mendes JF, Klafke GB, Ramis IB, Sanchotene KO, et al. Antifungal activity of diphenyl diselenide alone and in combination with itraconazole against Sporothrix brasiliensis. Med Mycol 2018b; (June):1-4.

19. Palomino J-C, Martin A, Camacho M, Guerra H, Swings J, Portaels F. Resazurin Microtiter Assay Plate: Simple and Inexpensive Method for Detection of Drug Resistance in Mycobacterium tuberculosis. Antimicrob Agents Chemother 2002; 46(8):2720 LP-2722.

20. Foerster S, Desilvestro V, Hathaway LJ, Althaus CL, Unemo M. A new rapid resazurin-based microdilution assay for antimicrobial susceptibility testing of Neisseria gonorrhoeae. J AntimicrobChemother2017;72(7): 1961-8.

21. Osaka I, Hefty PS. Simple Resazurin-Based Microplate Assay for Measuring Chlamydia Infections. Antimicrob Agents Chemother 2013; 57(6):2838 LP-2840. 